DOI:

\title{
NÚCLEO DE APOIO À CULTURA E À EXTENSÃO EM DIAGNÓSTICO ODONTOLÓGICO - RADIODIAGNÓSTICO EM ODONTOLOGIA
}

\author{
NUCLEOUS SUPPORT FOR CULTURE AND EXTENSION IN DIAGNOSIS \\ DENTISTRY - RADIODIAGNOSIS IN DENTISTRY
}

\author{
Plauto Christopher Aranha Watanabe ${ }^{1}$ \\ Elza Carneiro Santos ${ }^{2}$ \\ Vanessa de Araújo Faria ${ }^{3}$
}

\begin{abstract}
Resumo: O objetivo principal deste artigo é mostrar a importância das atividades desenvolvidas pelo Núcleo de Apoio às Atividades de Cultura e Extensão em Diagnóstico Odontológico (NACEDO), um Núcleo de Extensão da Pró-reitoria de Cultura e Extensão Universitária da Universidade de São Paulo (PRCEU/USP) como principal figura do tripé indissociável Extensão-Ensino-Pesquisa, para a Educação na Universidade. Métodologia: Através do atendimento de pacientes de rotina do Serviço de Radiodiagnóstico - NACEDO, onde circulam docentes, funcionários, alunos de graduação, pós-graduação, especialização/aprimoramento e, é claro, pacientes, referidos para realização de Diagnóstico em odontologia, trabalhamos as atividades fins da USP, indissociavelmente, fazendo chegar ao paciente e seus familiares, diversos aspectos de sua saúde geral. Resultados: Foram atendidos 13047 pacientes no período e realizados os respectivos laudos radiográficos, somados mais 250 laudos radiográficos realizados junto ao Hospital das Clínicas de Ribeirão Preto (HCRP). Todos esses pacientes, e/ou acompanhantes, com exceção dos pacientes do HCRP, responderam aos questionários e às orientações sobre a doença osteoporose. Conclusões: a interdisciplinaridade da atuação do NACEDO em Ribeirão Preto, através do Diagnóstico em odontologia trouxe um importante fenômeno de articulação do processo ensino-aprendizagem, teoria/prática, além de um rico material didático-científico ao grupo, e levou à comunidade informações inerentes à osteoporose, que é uma epidemia mundial. Resultado principal, são 15 anos de reflexão crítica conduzida pela Extensão Universitária.
\end{abstract}

Palavras-chave: Núcleo de Extensão. Diagnóstico Oral. Radiografia Panorâmica.

\footnotetext{
1 Professor Titular de Radiologia Odontológica, da Faculdade de Odontologia de Ribeirão Preto, da Universidade de São Paulo (FORP/USP). E-mail: watanabe@ forp.usp.br

2 Acadêmica do Curso de Pós-graduação Mestrado Profissional em Ciencias das Imagens e Física Médica, Diagnóstico por Imagem, da Faculdade de Medicina de Ribeirão Preto, da Universidade de São Paulo (USP). Email: santoselzacarneiro@gmail.com

${ }^{3}$ Acadêmica do Curso de Pós-graduação Diagnóstico Bucal, da Faculdade de Odontologia, da Universidade de São Paulo (USP). E-mail: vanessa.faria@usp.br
} 
Abstract: The main objective of this article is to show the importance of the activities developed by the Support Center for Culture and Extension Activities in Oral Diagnosis (NACEDO), an Extension Center of the Dean of Culture and University Extension of the University of São Paulo (PRCEU / USP) as the main figure of the inseparable tripod Extension-Teaching-Research, for Education at the University. Methodology: Through the care of routine patients of the Radiodiagnostic Service - NACEDO, where professors, employees, undergraduate and graduate students, specialization / improvement and, of course, patients referred to perform Diagnosis in dentistry, work with USP's core activities are inextricably linked, bringing different aspects of its general health to the patient and his family. Results: 13047 patients were treated during the period and the respective radiographic reports were made, plus another 250 radiographic reports made at the Ribeirão Preto Clinical Hospital (HCRP). All of these patients, and / or companions, with the exception of HCRP patients, answered questionnaires and guidelines on osteoporosis disease. Conclusions: the interdisciplinarity of NACEDO's performance in Ribeirão Preto, through Diagnosis in dentistry, brought an important phenomenon of articulation of the teaching-learning process, theory / practice, in addition to a rich didactic-scientific material to the group, and brought to the community inherent information osteoporosis, which is a worldwide epidemic. Main result is 15 years of critical reflection conducted by the University Extension.

Keywords: Extension Nucleus. Oral Diagnosis. Panoramic Radiography.

\section{Introdução}

Os Núcleos de Apoio às Atividades de Cultura e Extensão (NACEs) são órgãos de integração da Universidade de São Paulo (USP), instituídos com o objetivo de reunir docentes e especialistas, de um ou mais departamentos de uma Unidade ou de Unidades e Órgãos da Universidade, em torno de programas culturais ou de extensão, de caráter interdisciplinar e/ou de apoio instrumental à cultura e à extensão (RESOLUÇÃO COCEX Nº 6579, DE 19 DE JUNHO DE 2013)

O Núcleo de Apoio à Cultura e Extensão Universitária NACEDO, cujas sigla significa Núcleo de Apoio às Atividades de Cultura e Extensão em Diagnóstico Odontológico, foi instituído oficialmente pela resolução ${ }^{\circ}$ 5167, de 18 de novembro de 2004, com publicação no Diário Oficial do Estado de São Paulo (DOESP. 19.11.2004). Esse serviço foi uma evolução do extinto Serviço de Radiologia e Documentação Odontológica (SERDO) que funcionou até 2004 na Faculdade de Odontologia de Ribeirão Preto (FORP/USP). 


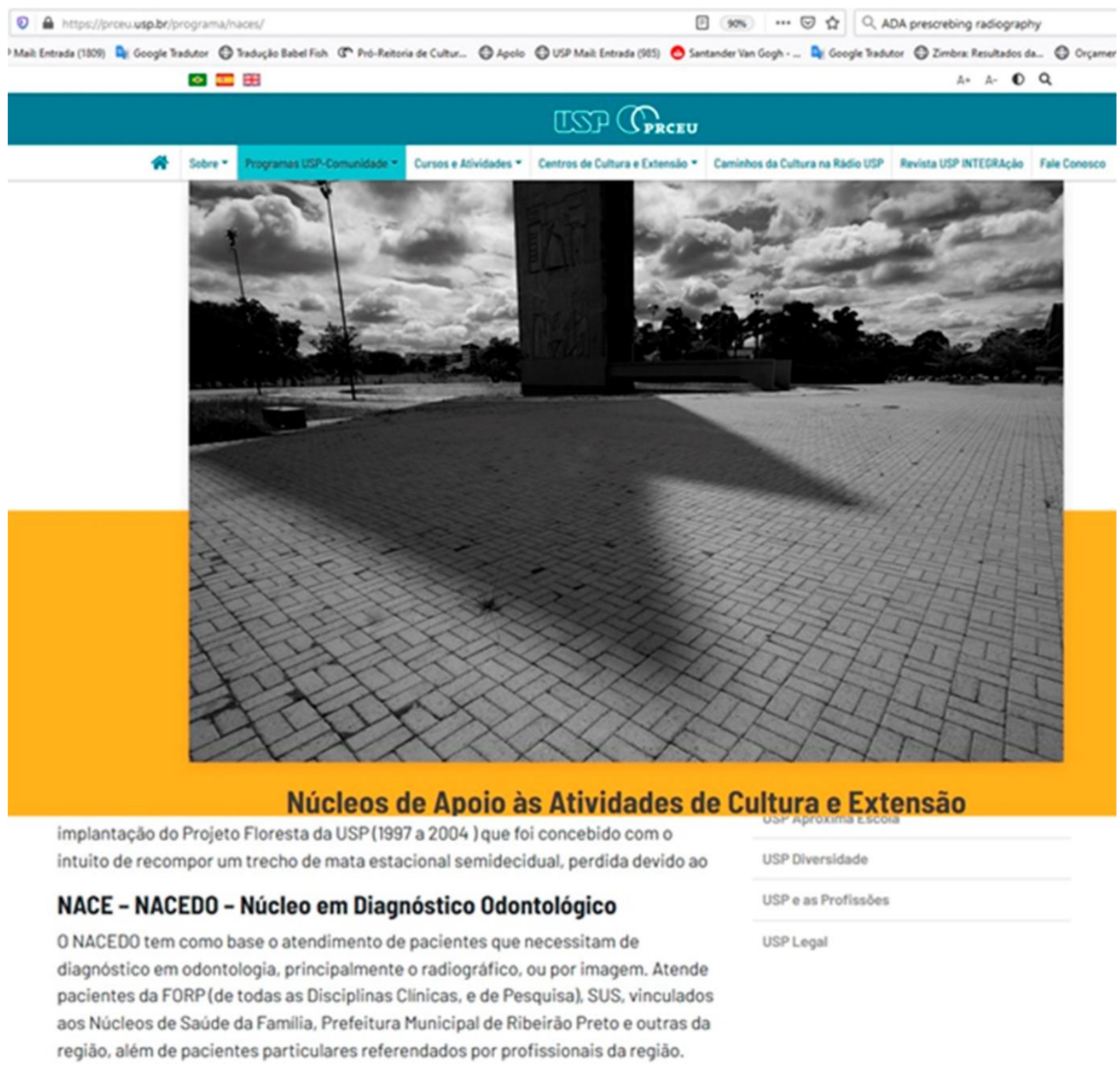

Figura 1: Foto da página da Pró-reitoria de Cultura e Extensão Universitária, da Universidade de São Paulo - PRCEU-USP, onde a universidade divulga seus Núcleos de Apoio às Atividades de Cultura e Extensão - NACEs à comunidade.

Fonte: Disponível em: https://prceu.usp.br/programa/naces/. Acesso em: 19 jul. 2020.

O NACEDO possui um objetivo comum com os outros núcleos da Universidade, articulando as ações de extensão com projetos, eventos, prestação de serviços, cursos, e publicações, que indissociavelmente articula ações de pesquisa e de ensino. Esse conjunto de ações contínuas de caráter educativo, social, cultural e científico/tecnológico procura alcançar um objetivo bem definido de avançar no desenvolvimento e na prevenção diagnóstica na Odontologia, e dentre seus objetivos estão: 
a) promover estudos sobre Diagnóstico Odontológico, em suas diversas especialidades;

b) fomentar o Diagnóstico para as várias especialidades clínicas da FORP/USP;

c) promover a atenção integral à saúde;

d) servir de base ao Sistema de Referência em Diagnóstico na Odontologia;

e) colaborar com organismos de saúde, sejam municipais, nacionais ou internacionais, com vistas a aprofundamentos e difusão de casos clínicos;

f) prestar assessoria e/ou consultoria a órgãos públicos e/ou organismos de saúde em assuntos relacionados ao diagnóstico odontológico;

g) difundir e participar de eventos que tenham por objeto de discussão questões relacionadas ao diagnóstico odontológico;

h) realizar pesquisas operacionais que possam contribuir com os estudos em desenvolvimento nos métodos avançados de diagnóstico.

Um correto diagnóstico nas diversas área das ciências da saúde é parte fundamental para se estabelecer um plano de tratamento adequado e, por isso, na Odontologia é um dos principais pilares que norteiam todo o tratamento odontológico.

Dentre os diversos exames complementares utilizados como auxílio ao diagnóstico odontológico, a radiografia panorâmica é rotineiramente utilizada, principalmente quando da primeira consulta do paciente. Desde 1994, a American Dental Association (ADA) implementou guidelines for prescribing dental radiographs, atualizado em 2012, que norteia a indicação radiográfica para diversas situações da saúde bucal, principalmente com base no exame clínico do paciente. Portanto, um dos principais passos do diagnóstico diz respeito à interpretação radiográfica das imagens obtidas (AMERICAN DENTAL ASSOCIATION (ADA); FOOD AND DRUG ADMINISTRATION (FDA), 2012).

$\mathrm{Na}$ rotina dos serviços médico-odontológicos que utilizam radiografias convencionais, a interpretação radiográfica é geralmente transcrita manualmente para o papel, por um profissional especializado. Com a entrada definitiva no mercado da radiologia digital, que permite a manipulação da imagem por meio da utilização de ferramentas e softwares, como brilho, contraste e aumento, os laudos radiográficos digitais se tornaram mais viáveis e, assim, são realizados em computadores, principalmente para facilitar a transcrição de termos, que 
podem ser digitados por auxiliares através de editores de texto, otimizando o tempo do profissional.

O desenvolvimento de novas tecnologias e métodos aplicados à ciência odontológica tem envolvido por demais o uso da computação. Soares et al. (1997) desenvolveram um software capaz de analisar os dados de interpretação radiográfica abrangendo, dentro da Odontologia, a área de Dentística. Uma ficha de interpretação radiográfica referente à experiência de cárie foi desenvolvida com a eleição das várias condições patológicas e anatômicas. Através do manuseio da linguagem de programação CLIPPER, essa ficha foi reproduzida, juntamente com uma análise estatística de pesquisa sobre todas e quaisquer condições relacionadas à experiência de cárie. Foram cadastrados os dados referentes aos 100 pacientes da FORP/USP.

Os resultados evidenciaram que a pesquisa desses dados, realizada pelo software, levou na época, cerca de 2 minutos e 33 segundos (1000\% mais rápida do que a realizada manualmente, fornecendo ainda toda uma análise estatística do assunto requerido). $\mathrm{O}$ desenvolvimento de uma ficha de pesquisa de interpretação radiográfica e um software, segundo os autores, agiliza infinitamente a pesquisa de dados referentes à experiência de cárie, trazendo também alta acuidade nos resultados.

Dessa forma, pretendemos mostrar a importância das atividades desenvolvidas pelo NACEDO, como principal figura do tripé indissociável Extensão-Ensino-Pesquisa, para a Educação na Universidade, por meio do Diagnóstico em Odontologia, para toda a comunidade dentro e fora da USP.

\section{Material e Métodos}

\section{Software ARI}

O Programa de Computador ARI - Análise Radiográfica Informatizada foi desenvolvido pelo NACEDO, em PHP (Hypertext Preprocessor), uma linguagem de programação interpretada no computador servidor, que funciona em conjunto com o programa Apache (WELCOME!, 2004) e Servidor HTTP. Esse programa é acessado pela intranet (rede local) ou internet, podendo-se utilizar qualquer navegador com suporte a HTML4 (Liguagem de marcação de hipertexto), Javascript (Linguagem de script executado no navegador do 
usuário) e CSS (Cascading Style Sheets - Linguagem de formatação). Os dados ficam registrados no programa de gerenciamento de banco de dados $M y S Q L$, um SGBD, ou seja, sistema de gerenciamento de banco de dados, que usa a linguagem SQL como interface.

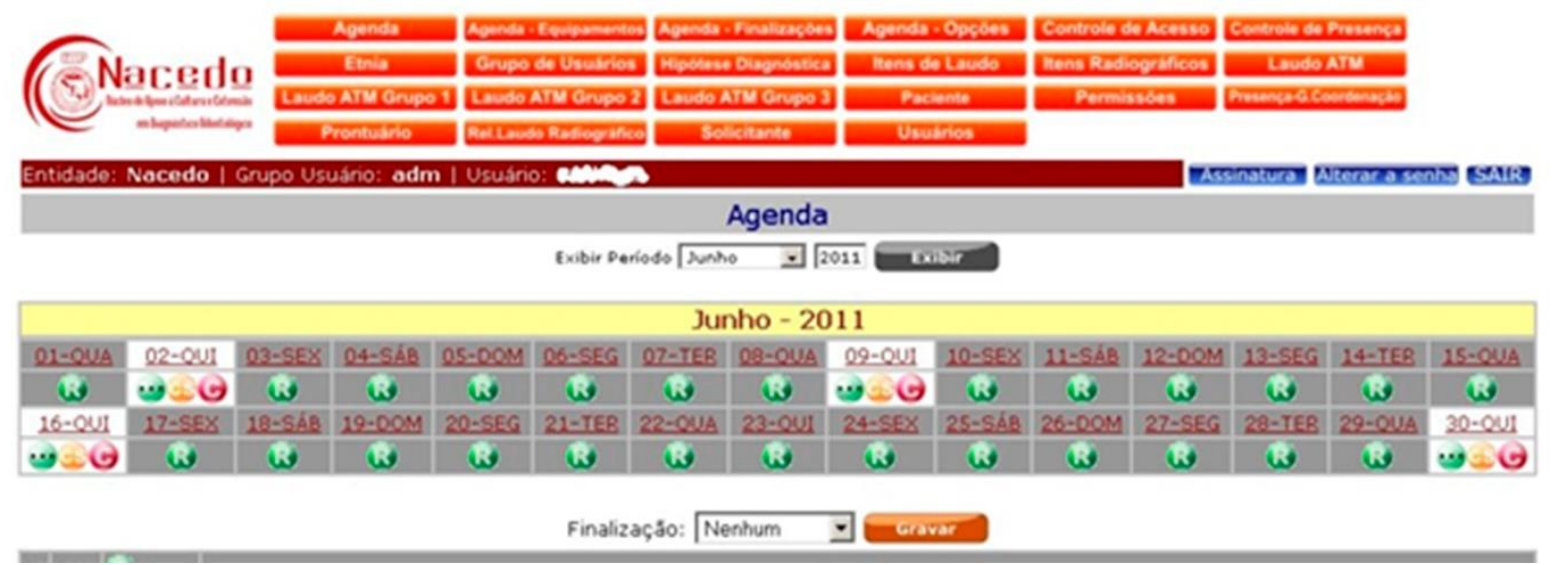

Figura 2: Tela do menu do site NACEDO, mostrando todas as possibilidades de utilização, inclusive da Agenda Radiográfica que controla o fluxo de pacientes, e também arquiva os principais dados dos pacientes, como nome, endereço, requisição radiográfica, data de nascimento, peso e altura.

Fonte: Elaboração da autoria.

O programa de computador ARI teve sua patente registrada na Revista de Propriedade Indústria sob $\mathrm{n}^{\mathrm{o}} 2039$, de 2 de fevereiro de 2010. Especificamente para as análises de interpretação radiográfica, foram desenvolvidas várias ferramentas para o Programa ARI para as diversas situações ou técnicas radiográficas referidas, principalmente, voltadas à técnica mais utilizada pelo cirurgião dentista para atendimento em primeira consulta, a radiografia panorâmica (AMERICAN DENTAL ASSOCIATION (ADA); FOOD AND DRUG ADMINISTRATION (FDA), 2012).

Por exemplo, o Software solicita e calcula de maneira automática o IMC (Índice de Massa Corporal), que fornece ao paciente e ao profissional uma medida rápida (utilizada internacionalmente) para calcular se a pessoa está no peso ideal. Essa medida foi desenvolvida por Lambert Quételet, no fim do século XIX, trata-se de um método fácil e rápido para a avaliação do nível de gordura de cada pessoa, sendo, por isso, um preditor internacional de obesidade adotado pela Organização Mundial da Saúde (WORLD HEALTH ORGANIZATION, 1994). 
A seleção de informações dinâmicas pré-definidas para os achados radiográficos e indicações de referência para o exame possibilitou uma maior confiabilidade no processo de Laudo Radiográfico (Fig. 3), estão listadas abaixo com suas respectivas funcionalidades nesse processo:

- radiografias utilizadas na interpretação: escolha de um ou mais itens, com opção de definir sua quantidade;

- laudo radiográfico resumido - a imagem radiográfica é compatível ou sugere: escolha de um ou mais itens informando os dentes relacionados;

- descrição de outros achados e da hipótese diagnóstica (H.D.);

- após o laudo ser finalizado, existe a opção "impressão" ou "retornar" para o laudo lançado;

- opção de localizar os laudos finalizados por nome do paciente, através de três opções de filtro ("iniciando", “que contenha", "finalizando") e opção de localizar por período da radiografia e/ou pelo nome do seu solicitante (Figura 4);

- impressão do laudo final através do navegador;

- ciência para o profissional, alunos e pacientes sobre as principais evidências de doença encontradas.

abrasão/atrição

acessórios metálicos colocados por razões cirúrgicas acessórios ortodônticos

alvéolos dentários em reparação

$\square$ ângulo mandibular acentuado

$\square$ anomalia

$\square$ anomalia de forma

$\square$ anomalia de tamanho

apinhamento dentário

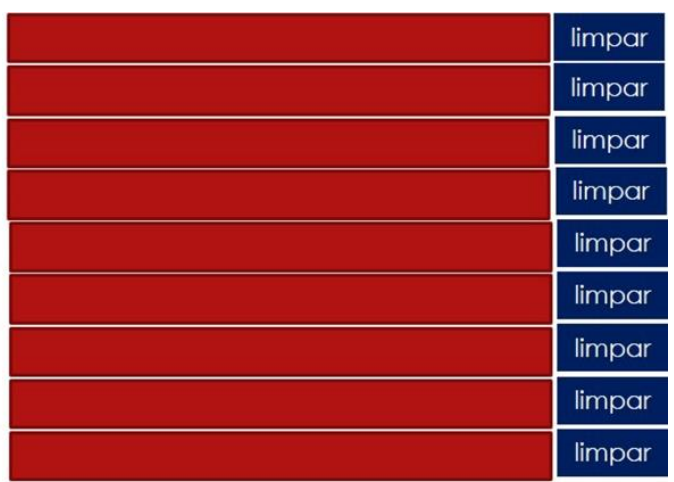

Figura 3: Parte dos itens de intrepretação radiográfica que o software oferece ao profissional, aluno ou docente para Laudar. A cada laudo, é possível colocar novos itens de interprteação radiográfica que ainda não constem.

Fonte: Elaboração da autoria. 


\section{O NACEDO na prática}

O serviço de radiodiagnóstico do NACEDO funciona dois períodos semanais para atendimento da população em geral referenciada para o serviço de radiodiagnóstico (vertente extensão), lotado na Faculdade de Odontologia de Ribeirão Preto, da Universidade de São Paulo (FORP/USP), na cidade de Ribeirão Preto/SP. Outros quatro períodos de funcionamento do NACEDO são destinados ao atendimento junto às disciplinas de graduação da FORP/USP, no Curso de Odontologia (vertente ensino). E outros dois períodos de atividades do NACEDO são reservados à pesquisa, que via de regra, envolve alunos de pósgraduação. Os outros períodos semanais remanescentes são ocupados com a parte administrativa, de radioproteção e de auxílio às outras disciplinas, aos cursos e às atividades fora dos muros da FORP/USP.

\section{Osteoporose na comunidade}

Todos os pacientes atendidos no NACEDO são convidados a responder a um questionário (Fig. 4), que avalia o risco de osteoporose. Essa ferramenta de análise é ministrada pelos alunos de graduação e/ou bolsistas de Iniciação Científica devidamente treinados e calibrados. Ao final do preenchimento e junto com o docente, rapidamente, o paciente é orientado sobre um possível risco avaliado no questionário a partir das informações de sua imagem radiográfica. Dessa forma, o paciente, em geral, é orientado a repassar para os seus familiares os questionamentos feitos no questionário e os aspectos negativos desse, bem como procurar por atendimento médico e rastreamento para a perda mineral óssea. 


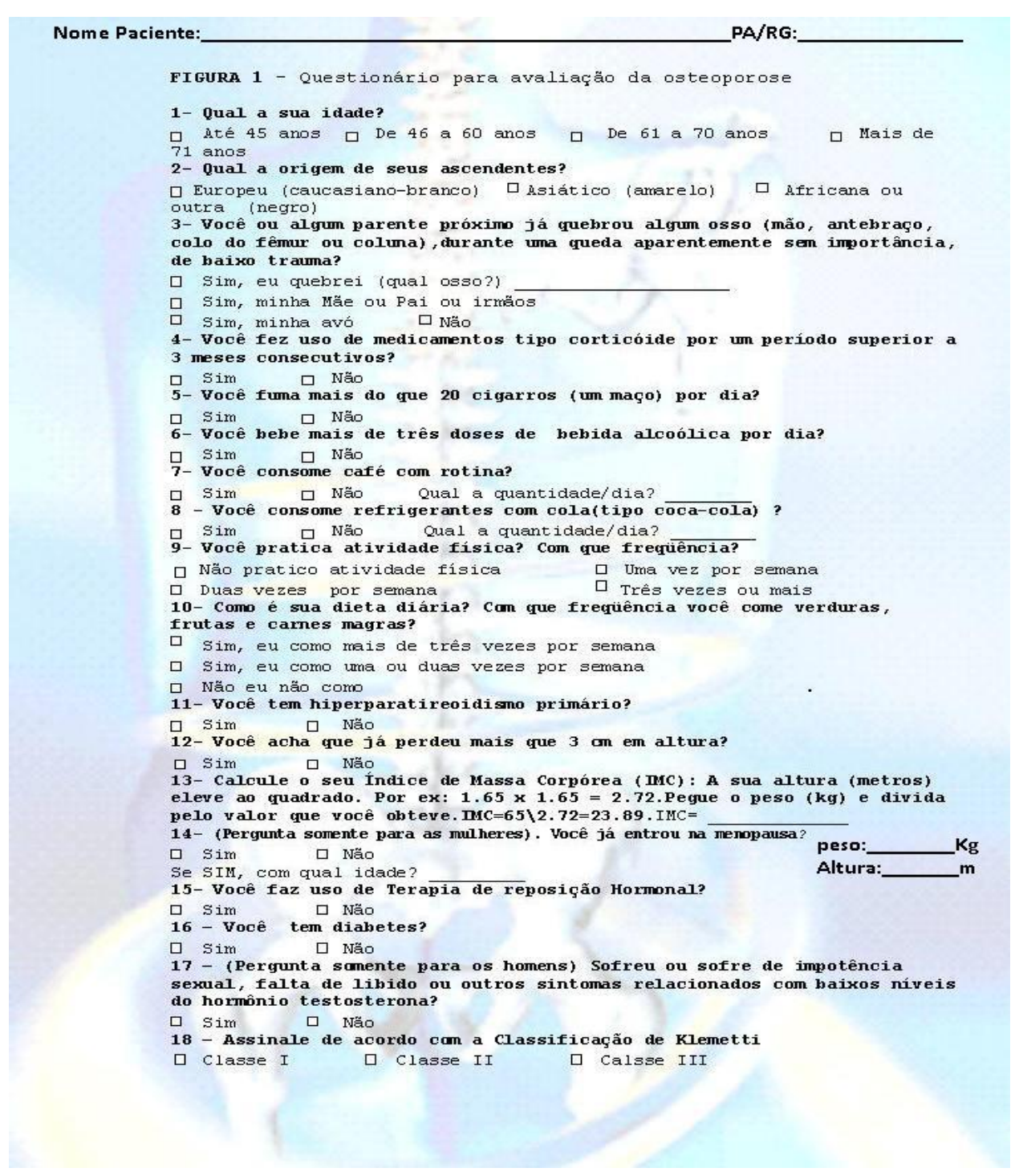

Figura 4: Questionário para avaliação do risco de fratura, osteoporose. Todos os pacientes que veem ao NACEDO respondem a essa ferramenta, e recebem esclarecimentos sobre os resultados, além da recomendação de leva-los aos familiares.

Fonte: NACEDO.

\section{Resultados}

O programa ARI foi utilizado por dois anos, na rotina de atendimento de pacientes no Serviço de Radiologia NACEDO, da Pró-Reitoria de Cultura e Extensão Universitária da USP, instalado na área de Radiologia da Faculdade de Odontologia FORP/USP, como avaliação piloto da ferramenta. Foi solicitada a patente em 2008 pela USP (Processo: 091712, publicado na Revista de Propriedade Industrial nº1993, p. 121, item 090, em 2010). 
Atualmente, possui 13047 (12 set. 2017) registros, ou seja, número correspondente aos atendimentos de pacientes que fizeram alguma imagem para diagnóstico, atendidos por algum profissional (cirurgião dentista) ou aluno de graduação/pós-graduação, sendo que o laudo se mostrou bastante confiável em seu propósito, disponibilizando dados para presentes e futuras estatísticas, com as mais variadas formas de resultados (Fig. 2 e 3) (Quadro 1). Os demais dados, até 2019, estão armazenados em sistema intrínseco da FORP, pois o site original foi invadido no final de 2017, fazendo com que a equipe se preocupasse mais com a segurança dos dados armazenados.

Quadro 1: Quantidade total de laudos realizados, por gênero e reabsorção de rebordos alveolares.

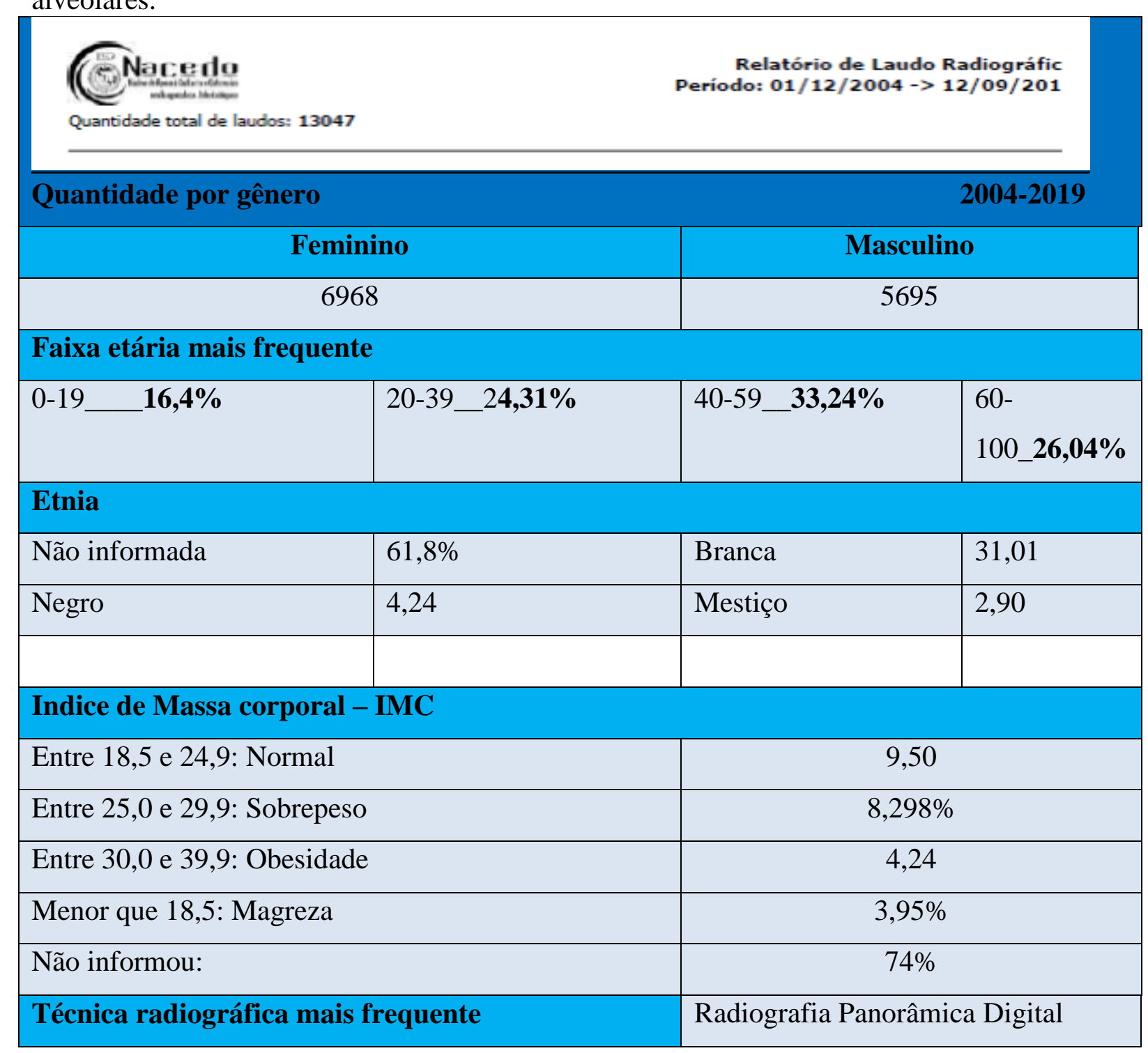




\begin{tabular}{|c|c|c|}
\hline Item de Laudo & Qtd. & $\%$ \\
\hline material restaurador radiopaco & 4030 & 8,936 \\
\hline imagem radiolúcida na coroa & 3071 & 6,809 \\
\hline dente(s) ausente(s) & 2705 & 5,990 \\
\hline reabsorção generalizada das cristas ósseas alveolares & 2312 & 5,126 \\
\hline canal(is) radicular(es)obturado(s) & 2155 & 4,778 \\
\hline cálculo(s) dentário(s) & 1570 & 3,481 \\
\hline dente(s) incluso(s) e/ou semincluso(s) e/ou impactado(s) & 1464 & 3.246 \\
\hline Demais estrut óss. complexo max.-mand. apres. aspecto de normalidade & 1446 & 3,206 \\
\hline Cabeca(s) da mandibula heteromorfo(s) & 1198 & 2,656 \\
\hline calcificasão da cadeia estilo-hioide & 1052 & 2,333 \\
\hline raiz residual & 1041 & 2,300 \\
\hline dente em formaçāo e/ou erup̧̧ão & 1007 & 2,233 \\
\hline indicada radiografia periapical & 381 & 1,953 \\
\hline imagem radiolúcida periapical circunscrita & 824 & 1,827 \\
\hline aumento do espaço pericementário & 823 & 1,825 \\
\hline imagem radiolúcida periapical difusa & 703 & 1.559 \\
\hline reabsorção da porção alveolar da maxila & 621 & 1,377 \\
\hline dente(s) deciduo(s) presente(s) no arco & 593 & 1,315 \\
\hline dilaceração radicular & 541 & 1.200 \\
\hline indicada radiografia interproximal & 530 & 1,175 \\
\hline cortical inferior mandibular Klemetti- Classe & 527 & 1,169 \\
\hline imagem radiolúcida contígua à restauração & 499 & 1,106 \\
\hline coros protética & 494 & 1,095 \\
\hline destruiçăo coronária & 485 & 1,075 \\
\hline aumento do espaço pericoronário & 469 & 1,040 \\
\hline acessónios ortoodônticos & 435 & 0,965 \\
\hline dente com éx́o de erupcão desfavorável & 411 & 0,911 \\
\hline envolvimento de furca & 409 & 0,907 \\
\hline pneumatizaç̃o do seio maxilar & 391 & 0,867 \\
\hline
\end{tabular}

Figura 5: Principais itens de laudo radiográfico identificados e suas respectivas porcentagens. Note que o primeiro item se refere à restauração (material restaurador radiopaco), podendo ser de metálica (amálgama) ou ainda plástica (resina radiopaca, mais moderna). O segundo item se refere, possivelmente, à cárie e/ou resina composta (mais antigo).

Fonte: Elaboração da autoria.

\section{Discussão}

A integralidade entre ensino, pesquisa e extensão, bem articulada, além da relação efetiva com a comunidade, objetiva conduzir a mudanças significativas nos processos de ensino e aprendizagem, colaborando efetivamente para a formação profissional de estudantes e professores, fortalecendo os atos de aprender, de ensinar e de formar profissionais e cidadãos.

A literatura sobre extensão é relativamente reduzida, e quando comparadas às correspondentes literaturas sobre ensino ou pesquisa, ela é discreta. Dentre elas, sobressaem diferentes conceitos de extensão universitária, principalmente referidas do livro de Botomé, de 1996, que foi Pró-Reitor de Extensão da Universidade Federal de São Carlos. Alguns vulgares como p.ex., "a Extensão como Prestação de Serviço"; enquanto a extensão como 
curso apoiou-se em ideias que foram importadas da Europa, ainda que interpretadas à nossa maneira; dos Estados Unidos da América, importaram-se a ideia de extensão como prestação de serviços sociais, adotada nos EUA também no final do século passado, aflorando no Brasil de uma maneira talvez diversa da original, ou seja, sem vínculos com o ensino e a pesquisa. Desta forma, a prestação de serviços sociais, sem vínculos com o ensino e a pesquisa seria, de acordo com alguns autores, extensão universitária. Outros, que pensam na mesma linha, consideram a extensão como instrumento de apoio às mudanças sociais (extensão como instrumento político-social), estando aqui apenas para que se confrontem com o anterior, mostrando que ele se insere num contexto um pouco mais amplo.

Essa ferramenta de integração do NACEDO, utilizando o software ARI produzido na rotina do NACEDO fomentou nos alunos, profissionais e docentes a extensão como processo educativo, cultural e científico que articula o Ensino e a Pesquisa de forma indissociável, e viabilizou a relação transformadora entre a Universidade e a sociedade, sobretudo, prevenindo os principais problemas de saúde da população e, dessa forma, contribuindo para a sua formação acadêmica, vivendo a realidade dentro e fora da Universidade. Os dados apresentados na Figura 5, por exemplo, ilustram que quase $16 \%$ de todos os pacientes atendidos tiveram experiência com cárie. Assim, podemos até apontar quais foram esses dentes mais atingidos por essa doença que ainda é o maior problema de saúde bucal no mundo.

A prestação de serviços à comunidade realizada no NACEDO mantém a indissociabilidade ensino-extensão-pesquisa, pois trata da criação e recriação de conhecimentos, que possibilita a transformação da sociedade, cuja questão central identificará o que deve ser pesquisado e para quais fins e interesses se buscam novos conhecimentos, como por exemplo, abordar doenças sistêmicas com esses pacientes atendidos, citamos uma doença que é uma epidemia mundial, negligenciada no Brasil, a osteoporose (WATANABE, 2007; ANDRADE, 2011), devido às características encontradas em uma imagem radiográfica bucal. Todos os pacientes assistidos no Serviço de Radiodiagnóstico do NACEDO, lotado na FORP, possuem exames por imagem e, também, laudos de interpretação diagnóstica nas mais diversas requisições arquivados em computador, no software ARI, além dos dados colhidos nos questionários sobre risco de fratura/osteoporose. 
Os temas radiografia panorâmica, diagnóstico e doenças sistêmicsa são assuntos essenciais do currículo de qualquer curso de odontologia, assim, a extensão de serviços à comunidade, aplicando a prática desses temas, propiciou ao aluno participar da melhoria das condições de vida da sociedade articulando as principais necessidades de pesquisa, com base na política do SUS. Um exemplo para essa evidência é a avaliação de risco da doença osteoporose, epidemia mundial, identificada em radiografias panorâmicas e questionários de avaliação de risco dessa doença. Ainda não temos retorno sobre o procedimento dos pacientes levarem aos seus familiares os aspectos inerentes ao risco avaliado nos questionários, mas pretendemos fazer isso, integrando alunos e/ou conhecimentos nos grupos que participam dos Núcleos de Saúde da Família.

Várias disciplinas estão envolvidas mais intrinsecamente no desenvolvimento do Projeto, que são: Radiologia Básica; Estomatologia; Diagnóstico Clínico Integrado e Diagnóstico I e II.

A Osteoporose foi definida pela Organização Mundial da Saúde (OMS), como uma doença caracterizada por baixa densidade mineral óssea e deterioração da microarquitetura do tecido ósseo, conduzindo a sua fragilidade e um consequente aumento do risco de fratura (BAIM; LESLIE, 2012; KANIS et al., 2002).

As radiografias panorâmicas assim como as periapicais são amplamente utilizadas na clínica odontológica como exames complementares pela facilidade de acesso e baixo custo. A radiografia panorâmica sofreu nas últimas décadas excelente desenvolvimento técnico, tornou-se mais acurada e definida, o que permitiu seu uso cada vez mais amplo na Odontologia.

A perda de massa óssea na região oral é uma das principais causas de morbidade na população da terceira idade (VAISHNAV et al., 2010). Vários estudos têm sido realizados sobre a utilidade de radiografias panorâmicas no rastreio da osteoporose (má qualidade óssea) em clínicas odontológicas (TAGUCHI et al., 1999; 2007). A erosão da cortical da mandíbula detectada em radiografias panorâmicas pode ser útil na identificação de mulheres com osteoporose (KLEMETTI et al., 1993; TAGUCHI et al., 2007).

Sendo assim, o objetivo da avaliação não se baseia no diagnóstico específico da osteoporose, mas sim consiste em identificar possível correlação dos achados na radiografia panorâmica, por meio de exames realizados rotineiramente nos consultórios. 
Instruindo/esclarecendo o paciente sobre a doença, utilizando o questiuonário como a avaliação do risco à fratura, e este levando essa situação aos familiares, poderemos estar, claramente, realizando uma atividade puramente extensionista, mas que ainda não foi avaliada, e isto levará tempo.

O Núcleo de Extensão (NACEDO) é privilegiado para a pesquisa, especialmente a pesquisa-ação que, na própria concepção, prevê a perspectiva de transformação do contexto em que se trabalha. Assim, essas atividades em diagnóstico do NACEDO puderam identificar os principais sinais radiográficos presentes nas cavidades bucais relacionados ou não às principais doenças sistêmicas da população, possibilitando a transformação do ensino, da prática odontológica e da priorização de pesquisas na área da saúde.

\section{Conclusões}

O NACEDO, lotado no Campus USP-Ribeirão Preto, trabalhou durante 15 anos, ininterruptamente, com recursos próprios (conditio sine qua non para sua aprovação e funcionamento), interdisciplinaridade de atuação com base no tripé da USP, Extensão, Ensino e Pesquisa, trazendo um importante fenômeno de articulação do processo ensinoaprendizagem, teoria/prática, das disciplinas de graduação, pós-graduação e disciplinas de cursos de extensão. Esperamos que os alunos possam em seus novos ambientes, no decorrer do processo, pela interdisciplinaridade, pela relação de reciprocidade e de mutualidade, pressupondo uma atitude diferente a ser assumida frente ao problema de conhecimento e trocando a concepção fragmentária para uma concepção de unidade do Diagnóstico do ser humano", devolver à comunidade toda essa singularidade criada entorno do Núcleo de Apoio à Cultura e Extensão em Diagnóstico Odontológico.

Esse trabalho, no NACEDO com os pacientes, alunos e profissionais, sempre integrou, com o diálogo e disponibilidade como opções, a melhoria da interdisciplinaridade e as partes, e cremos, portanto, que provocou a transformação da própria realidade da comunidade. 


\section{Referências}

AMERICAN DENTAL ASSOCIATION (ADA); FOOD AND DRUG ADMINISTRATION (FDA). Council on scientific affairs. [S. l.], n. ADA Positions, Policies and Statements, p. 127, 2012. Disponível em: http://www.ada.org/ /media/ADA/Member

Center/FIles/Dental_Radiographic_Examinations_2012.ashx.

ANDRADE, K. M. de; CHEADE, M. de C. C.; ALONSO, M. B. C. C. et al. Avaliação do risco à osteopenia/osteoporose através do questionário avaliativo e sua associação com a análise morfológica do córtex inferior da mandíbula realizadas em radiografias panorâmicas. Revista Paulista de Odontologia, São Paulo, v. 33, p. 47-53, abr./mai./jun. 2011.

ASSESSMENT OF FRACTURE RISK AND ITS APPLICATION TO SCREENING FOR POSTMENOPAUSAL OSTEOPOROSIS. Report of a WHO Study Group. Geneva, World Health Organization, 1994. (WHO Technical Report Series, n. 843).

BAIM, S.; LESLIE, W. D. Assessment of fracture risk Current Osteoporosis Reports, v. 10, p. 28-41, 2012.

BATOMÉ, S. P. Pesquisa alienada e ensino alienante: o equívoco da extensão universitária. Petrópolis: Vozes, 1994.

KANIS, J. A. el al. A new approach to the development of assessment guidelines for osteoporosis. Osteoporosis International, [ S.1], v. 13, n. 7, p. 527-536, 2002.

KLEMETTI, E.; VAINIO, P.; LASSILA, V.; ALHAVA, E. Cortical bone mineral density in the mandible and osteoporosis status in postmenopausal women. European Journal of Oral Sciences, [ s. 1], v. 101, n.4, p. 219-223, 1993.

SÃO PAULO. Resolução COCEX no 6579, de 19 de junho de 2013. Disponível em: https://prceu.usp.br/wp-content/uploads/2015/01/RESOLUCAO_6579.pdf.

SOARES, M. R.; WATANABE, P. C. A.; PARDINI, L. C.; GOMES, C. C.; ARITA, E. S. "Software" para pesquisa de dados de interpretação radiográfica referente à experiência de cárie. RPG, v. 4, n. 3, p.197-200, jul./ago./set. 1997.

TAGUCHI, A; OHTSUKA, M.; TSUDA, M.; NAKAMOTO, T.; KODAMA, I.; INAGAKI, K.; NOGUCHI, T.; KUDO, Y.; SUEI, Y.; TANIMOTO, K. Risk of vertebral osteoporosis in post-menopausal women with alterations of the mandible. Dentomaxillofacial Radiology, v. 36, p. 143-48, 2007.

VAISHNAV, K.; SHAH, D.; PATEL, P. A panoramic evaluation of the mandibular canal wall resorption in relation to Diabetes, Thyroid and Asthma in edentulous patients. Journal of International Oral Health, Gujarat, v. 2, n. 3, p. 27-32, out. 2010. 
WATANABE, P. C. A.; ISSA, J. P. M.; OLIVEIRA, T. M.; MONTEIRO, S. A. C.; IYOMASSA, M. M.; REGALO, S. C. H.; SIESSERE, S. Morphological study of the mandibular trabecular bone in panoramic radiographs. International Journal of Morphology, v. 25, p. 875-880, 2007.

WELCOME! The Apache HTTP Server Project. Disponível em: http://httpd.apache.org/.

Recebido em: 28 de março de 2018.

Aceito em: 20 de julho de 2020. 